On the facial structure of the set of correlation matrices

M. Laurent and S. Poljak

Department of Operations Reasearch, Statistics, and System Theory

BS-R9501 1995 
Report BS-R9501

ISSN 0924-0659

CWI

P.O. Box 94079

1090 GB Amsterdam

The Netherlands

$\mathrm{CWI}$ is the National Research Institute for Mathematics and Computer Science. CWI is part of the Stichting Mathematisch Centrum (SMC), the Dutch foundation for promotion of mathematics and computer science and their applications.

SMC is sponsored by the Netherlands Organization for Scientific Research (NWO). CWI is a member of ERCIM, the European Research Consortium for Informatics and Mathematics.

Copyright @ Stichting Mathematisch Centrum P.O. Box 94079, 1090 GB Amsterdam (NL) Kruislaan 413, 1098 SJ Amsterdam (NL) Telephone +3120 5929333 Telefax +3120 5924199 


\title{
On the Facial Structure of the Set of Correlation Matrices
}

\author{
Monique Laurent ${ }^{1}$ \\ $C W I$ \\ P.O. Box 94079 \\ 1090 GB Amsterdam \\ The Netherlands
}

\author{
Svatopluk Poljak ${ }^{2}$ \\ Fakultät für Mathematik und Informatik \\ Universität Passau \\ Innstrasse 33, 94030 Passau \\ Germany
}

\begin{abstract}
We study the facial structure of the set $\mathcal{E}_{n \times n}$ of correlation matrices (i.e., the positive semidefinite matrices with diagonal entries equal to 1 ). In particular, we determine the possible dimensions for a face, as well as for a polyhedral face of $\mathcal{E}_{n \times n}$. It turns out that the spectrum of face dimensions is lacunary and that $\mathcal{E}_{n \times n}$ has polyhedral faces of dimension up to $\approx \sqrt{2 n}$. As an application, we describe in detail the faces of $\mathcal{E}_{4 \times 4}$. We also discuss results related to optimization over $\mathcal{E}_{n \times n}$.

AMS Subject Classification (1991): 15A57, 52A37, 90C27.

Keywords and Phrases: correlation matrix, convex set, normal cone, face, polytope, dimension, Laplacian matrix, max-cut.
\end{abstract}

\section{Introduction}

A positive semidefinite matrix whose diagonal entries are equal to 1 is called a correlation matrix. Let $\mathcal{E}_{n \times n}$ denotes the set of $n \times n$ correlation matrices, i.e.,

$$
\mathcal{E}_{n \times n}:=\left\{X \in \mathbb{R}^{n \times n} \mid X \succeq 0, x_{i i}=1 \text { for all } i=1, \ldots, n\right\} .
$$

The notation $X \succeq 0$ means that $X$ is a symmetric positive semidefinite matrix. The convex set $\mathcal{E}_{n \times n}$ is called the elliptope. Let us recall two previously known results that are also crucial for this paper.

\footnotetext{
${ }^{1}$ On leave from LIENS, Ecole Normale Supérieure, Paris

${ }^{2}$ The research was partly done while the author visited CWI, Amsterdam, with a grant from the Stieltjes Institute, whose support is gratefully acknowledged
} 
TheOREM 1.1 [LT94] Let $A \in \mathcal{E}_{n \times n}$ be a correlation matrix of rank $r$ and let $F(A)$ be the smallest face of $\mathcal{E}_{n \times n}$ containing $A$. Then,

$$
\operatorname{dim} F(A)=\left(\begin{array}{c}
r+1 \\
2
\end{array}\right)-\operatorname{rank}\left(v_{i} v_{i}^{T} \mid 1 \leq i \leq n\right) .
$$

where $v_{1}, \ldots, v_{n} \in \mathbb{R}^{r}$ is a collection of vectors such that $A=\operatorname{Gram}\left(v_{1}, \ldots, v_{n}\right)$.

Theorem 1.1 generalizes results of [CM79, Loe80, GPW90], where was mainly considered the question of determining the possible ranks for extreme elements of $\mathcal{E}_{n \times n}$. The elliptope is a nonpolyhedral convex set and has a nonsmooth boundary. The points $X \in \mathcal{E}_{n \times n}$ with full dimensional normal cone are called vertices.

TheOREM 1.3 [LP93] The elliptope $\mathcal{E}_{n \times n}$ has precisely $2^{n-1}$ vertices, each of the form $a a^{T}$ for $a \in\{-1,1\}^{n}$.

Theorem 1.3 was motivated by the fact that $\mathcal{E}_{n \times n}$ is a relaxation of a hard combinatorial optimization problem, namely, the max-cut problem. Indeed, the rank one matrices of $\mathcal{E}_{n \times n}$ are of the form $a a^{T}$ for $a \in\{-1,1\}^{n}$; they are called cut matrices as they correspond to the cuts of the complete graph. The convex hull of the cut matrices defines a polytope, called the cut polytope and denoted by $\mathrm{CUT}_{n \times n}$. Then, the max-cut problem is the problem of optimizing a linear objective function over the cut polytope. Hence, $\mathcal{E}_{n \times n}$ can be seen as a (nonpolyhedral) relaxation of the cut polytope (see [LP93, La94]). Moreover, a recent result of [GW94] shows that by optimizing over the elliptope one obtains a very good approximation for the max-cut problem.

Some other papers [GJSW84, BJT93, La94] study the projection $\mathcal{E}(G)$ of $\mathcal{E}_{n \times n}$ on the edge set of a graph $G$; this corresponds to the question of determining what partial matrices can be completed to a positive semidefinite matrix.

The subject of this paper is the facial structure of the elliptope $\mathcal{E}_{n \times n}$. Section 2 contains several old and new preliminary results. In Section 3 , we describe all possible values for the dimension of a face of $\mathcal{E}_{n \times n}$. We show that for all 'admissible' values $k$ within the range of (1.2), there exists a face of dimension $k$. Our further results from Section 4 concern the polyhedral faces of $\mathcal{E}_{n \times n}$. A polyhedral face is, in some sense, the most 'nonsmooth part' of the boundary of $\mathcal{E}_{n \times n}$. We determine the largest possible dimension for a polyhedral face and we show that it can be realized by a simplex face whose vertices are cut matrices. In Section 5 , we group some results related to optimization over the elliptope. In particular, we present a link between the faces of the elliptope and the dimension of the optimized eigenspace in the dual problem. Finally, we treat in detail in Section 6 the elliptope $\mathcal{E}_{4 \times 4}$; the elliptope $\mathcal{E}_{3 \times 3}$ having been described in [LP93]. We describe the proper faces of $\mathcal{E}_{4 \times 4}$, whose possible dimensions are $0,1,2$ and 3 ; faces of dimension 1 are edges between two cut matrices and faces of dimension 3 are ismorphic to $\mathcal{E}_{3 \times 3}$. The highest dimension for a polyhedral face of $\mathcal{E}_{4 \times 4}$ is 2 . 


\section{Old and new basic facts}

We start with some well known facts, formulated in the following two lemmas.

Lemma 2.1 Let $x_{1}, \ldots, x_{n}$ be $n$ linearly independent vectors in $\mathbb{R}^{n}$. Then, the system

$$
\mathcal{S}:=\left\{x_{i} x_{i}^{T} \mid 1 \leq i \leq n\right\} \cup\left\{\left(x_{i}-x_{j}\right)\left(x_{i}-x_{j}\right)^{T} \mid 1 \leq i<j \leq n\right\}
$$

is linearly independent.

Proof. As $\mathcal{S}$ consists of $n+\left(\begin{array}{c}n \\ 2\end{array}\right)=\left(\begin{array}{c}n+1 \\ 2\end{array}\right)$ elements, it suffices to show that, if $X$ is a symmetric $n \times n$ matrix orthogonal to all members of $\mathcal{S}$, then $X$ is the zero matrix. By assumption, $\left\langle X, x_{i} x_{i}^{T}\right\rangle=x_{i}^{T} X x_{i}=0$ for $i=1, \ldots, n$, and $\left\langle X,\left(x_{i}-x_{j}\right)\left(x_{i}-x_{j}\right)^{T}\right\rangle=\left(x_{i}-x_{j}\right)^{T} X\left(x_{i}-x_{j}\right)=0$, implying that $x_{i}^{T} X x_{j}+$ $x_{j}^{T} X x_{i}=0$ for $1 \leq i<j \leq n$. We check that $x^{T} X x=0$ for all $x \in \mathbb{R}^{n}$. Indeed, let $x=\sum_{1 \leq i \leq n} \alpha_{i} x_{i}$ for some scalars $\alpha_{i}$. Then, $x^{T} X x=\sum_{1 \leq i \leq n} \alpha_{i}^{2} x_{i}^{T} X x_{i}+$ $\sum_{1 \leq i<j \leq n} \alpha_{i} \alpha_{j}\left(x_{i}^{T} X x_{j}+x_{j}^{T} X x_{i}\right)=0$. This implies that $X=0$; indeed, if $x$ is an eigenvector of $X$ for the eigenvalue $\lambda$, then $0=x^{T} X x=\lambda\|x\|^{2}$, yielding $\lambda=0$.

The Gram matrix $\operatorname{Gram}\left(v_{1}, \ldots, v_{k}\right)$ of a collection of vectors $v_{1}, \ldots, v_{k}$ is the $k \times k$ symmetric matrix whose $(i, j)$-th entry is equal to $v_{i}^{t} v_{j}$. The linear subspace spanned by vectors $v_{1}, \ldots, v_{k}$ is denoted $\left\langle v_{1}, \ldots, v_{k}\right\rangle$.

Lemma 2.2 Let $v_{1}, \ldots, v_{k} \in \mathbb{R}^{n}$. Then

$$
\operatorname{dim}\left(\left\langle v_{1}, \ldots, v_{k}\right\rangle\right)=\operatorname{rank}\left(\operatorname{Gram}\left(v_{1}, \ldots, v_{k}\right)\right)=\operatorname{rank}\left(\sum_{i=1}^{k} v_{i} v_{i}^{t}\right) .
$$

In the whole paper, when dealing with matrices, we take as ambient space the set of symmetric matrices equipped with the inner product

$$
\langle A, B\rangle:=\operatorname{Tr}(A B)=\sum_{1 \leq i, j \leq n} a_{i j} b_{i j}
$$

\subsection{The kernel of a correlation matrix}

It is easy to see that 
LEMma 2.3 The relative interior of $\mathcal{E}_{n \times n}$ consists of the positive definite correlation matrices and its relative boundary of the correlation matrices $X$ with $\operatorname{rank}(X)<n$.

Let $X \in \mathcal{E}_{n \times n}$. Clearly, each nonzero vector of $\operatorname{ker}(X)$ has at least two nonzero coordinates. It is shown in [DP93b] that every vector $v \in \operatorname{ker}(X)$ is balanced, i.e., satisfies

$$
\left|v_{i}\right| \leq \sum_{1 \leq j \leq n, j \neq i}\left|v_{j}\right| \text { for all } i=1, \ldots, n
$$

TheOREM 2.4 [DP93b] Given a vector $v \in \mathbb{R}^{n}$, there exists a correlation matrix $X \in \mathcal{E}_{n \times n}$ such that $X v=0$ if and only if $v$ is balanced.

Note that there exist balanced vectors $v \in \mathbb{R}^{n}$ for which there exists no matrix $X \in \mathcal{E}_{n \times n}$ for which equality $\operatorname{ker}(X)=\langle v\rangle$ holds. This is the case, for instance, for the vector $v=(n, 1, \ldots, 1)$; see Theorem 2.6. Call a vector $v \in \mathbb{R}^{n}$ strictly balanced if it satisfies

$$
\left|v_{i}\right|<\sum_{1 \leq j \leq n, j \neq i}\left|v_{j}\right| \text { for all } i=1, \ldots, n .
$$

Lemma 2.5 Let $X \in \mathcal{E}_{n \times n}$ with $\left|x_{i j}\right|<1$ for all $i \neq j$. Then, every nonzero vector $v \in \operatorname{ker}(X)$ is strictly balanced.

Proof. Suppose that $\left|v_{1}\right|=\left|v_{2}\right|+\ldots+\left|v_{n}\right|$. From $X v=0$, we obtain that $\sum_{2 \leq i \leq n} x_{1 i} v_{i}=-v_{1}$. Therefore,

$$
\left|v_{1}\right|=\left|\sum_{2 \leq i \leq n} x_{1 i} v_{i}\right| \leq \sum_{2 \leq i \leq n}\left|x_{1 i}\right|\left|v_{i}\right| \leq \sum_{2 \leq i \leq n}\left|v_{i}\right|=\left|v_{1}\right| .
$$

Hence, equality holds throughout, which implies that $\sum_{2 \leq i \leq n}\left(\left|x_{1 i}\right|-1\right)\left|v_{i}\right|=0$. Therefore, $v_{2}=\ldots=v_{n}=0$, a contradiction.

Theorem 2.6 Let $v \in \mathbb{R}^{n}$ such that $v_{i} \neq 0$ for all $i$. Then, the following statements are equivalent.

(i) There exists $X \in \mathcal{E}_{n \times n}$ such that $\operatorname{ker}(X)=\langle v\rangle$.

(ii) The vector $v$ is strictly balanced.

Proof. $(i) \Longrightarrow(i i)$ Let $X \in \mathcal{E}_{n \times n}$ such that $\operatorname{ker}(X)=\langle v\rangle$. Then, $\left|x_{i j}\right|<1$ for all $i \neq j$. (If, say, $x_{12}=1$, then the vector $(1,-1,0 \ldots, 0)$ belongs to $\operatorname{ker}(X)$; hence, 
it coincides with $v$, which contradicts the fact that all entries of $v$ are nonzero.) Therefore, $v$ is strictly balanced by Lemma 2.5 .

(ii) $\Longrightarrow(i)$ We follow partly the proof of (Theorem 3.2, [DP93b]). We can suppose without loss of generality that $v_{1}, \ldots, v_{n}>0$. For $h=1, \ldots, n$, set

$$
1+\epsilon_{h}:=\left(\frac{\sum_{i \neq h} v_{i}}{v_{h}}\right)^{2}
$$

then, $\epsilon_{h}>0$. Define the vector

$$
x_{h}:=\left(1, \ldots, 1, \sqrt{1+\epsilon_{h}}, 1, \ldots, 1\right) \in \mathbb{R}^{n},
$$

where $\sqrt{1+\epsilon_{h}}$ stands at the $h$-th position. Set also

$$
t:=\frac{\sum_{1 \leq h \leq n} \frac{1}{\epsilon_{h}}}{1+\sum_{h} \frac{1}{\epsilon_{h}}}, \quad \alpha_{h}:=\frac{1-t}{\epsilon_{h}} \quad \text { for } h=1, \ldots, n .
$$

Finally, let

$$
X:=\sum_{1 \leq h \leq n} \alpha_{h} x_{h} x_{h}^{T} .
$$

Clearly, $X \succeq 0$ as $\alpha_{h}>0$ since $0<t<1$. One can check that the diagonal entries of $X$ are equal to 1 . Moreover, $X v=0$ since $v$ is orthogonal to $x_{1}, \ldots, x_{n}$ and $\operatorname{ker}(X)=\langle v\rangle$ as the rank of $X$ is equal to the rank of $\left\{x_{1}, \ldots, x_{n}\right\}$, i.e., to $n-1$ (see Lemma 2.2).

Note that Thoerem 2.6 does not hold if some entries of $v$ are equal to 0 . For instance, the vector $v=(0,1,1)$ is not strictly balanced but the kernel of the matrix

$$
\left(\begin{array}{ccc}
1 & 1 / 2 & -1 / 2 \\
1 / 2 & 1 & -1 \\
-1 / 2 & -1 & 1
\end{array}\right)
$$

is spanned by $v$.

\subsection{Faces}

A subset $F$ of a convex set $K$ is called a face (or extreme set) of $K$ if, for all $x \in F, y, z \in K, 0 \leq \alpha \leq 1, x=\alpha y+(1-\alpha) z$ implies that $y, z \in F$. We recall some facts, taken from [LP93], on the faces of $\mathcal{E}_{n \times n}$.

Theorem 2.7 [LP93] For every subspace $V$ of $\mathbb{R}^{n}$, the set

$$
F_{V}:=\left\{X \in \mathcal{E}_{n \times n} \mid \operatorname{ker}(X) \supseteq V\right\}
$$


is a face of $\mathcal{E}_{n \times n}$. Conversely, every face $F$ of $\mathcal{E}_{n \times n}$ is of the form $F_{V}$, where $V=\bigcap_{X \in F} \operatorname{ker}(X)$. In particular, given $X_{0} \in \mathcal{E}_{n \times n}$, let $F\left(X_{0}\right)$ denote the smallest face of $\mathcal{E}_{n \times n}$ that contains $X_{0}$. Then,

$$
F\left(X_{0}\right)=\left\{X \in \mathcal{E}_{n \times n} \mid \operatorname{ker}(X) \supseteq \operatorname{ker}\left(X_{0}\right)\right\} .
$$

Faces of $\mathcal{E}_{n \times n}$ can be "lifted" to faces of $\mathcal{E}_{(n+1) \times(n+1)}$ (of the same dimension) in the following way. Let $X$ be a symmetric $n \times n$ matrix with diagonal entries equal to 1 , of the form

$$
X=\left(\begin{array}{l|l}
Y & a \\
\hline a^{t} & 1
\end{array}\right)
$$

where $a \in \mathbb{R}^{n-1}$ and $Y$ is a symmetric $(n-1) \times(n-1)$ matrix. Consider the $(n+1) \times(n+1)$ symmetric matrices $X^{\prime}$ and $X^{\prime \prime}$ defined by

$$
X^{\prime}=\left(\begin{array}{c|c|c}
Y & a & a \\
\hline a^{t} & 1 & 1 \\
\hline a^{t} & 1 & 1
\end{array}\right), X^{\prime \prime}=\left(\begin{array}{c|c|c}
Y & a & -a \\
\hline a^{t} & 1 & -1 \\
\hline-a^{t} & -1 & 1
\end{array}\right) .
$$

For a subset $F$ of $\mathcal{L}_{n}$, set $F^{\prime}:=\left\{X^{\prime} \mid X \in F\right\}$ and $F^{\prime \prime}:=\left\{X^{\prime \prime} \mid X \in F\right\}$. Then,

$$
X \in \mathcal{L}_{n} \Longleftrightarrow X^{\prime} \in \mathcal{L}_{n+1} \Longleftrightarrow X^{\prime \prime} \in \mathcal{L}_{n+1},
$$

$F$ is a face of $\mathcal{L}_{n} \Longleftrightarrow F^{\prime}$ is a face of $\mathcal{L}_{n+1} \Longleftrightarrow F^{\prime \prime}$ is a face of $\mathcal{L}_{n+1}$.

Clearly, $F, F^{\prime}$ and $F^{\prime \prime}$ all have the same dimension. We say that $F^{\prime}, F^{\prime \prime}$ are liftings of the face $F$. Moreover, if $F$ is a face of $\mathcal{E}_{n \times n}$ and $V=\bigcap_{X \in F} \operatorname{ker}(X)$, then the subspace $V^{\prime}:=\bigcap_{Y \in F^{\prime}} \operatorname{ker}(Y)$ is generated by the vectors $(v, 0)(v \in V)$ and $(0, \ldots, 0,1,-1)$, while the subspace $V^{\prime \prime}:=\bigcap_{Y \in F^{\prime \prime}} \operatorname{ker}(Y)$ is generated by the vectors $(v, 0)(v \in V)$ and $(0, \ldots, 0,1,1)$. The following result permits to recognize if a face arises as a lifting of another face.

Lemma 2.8 Let $F$ be a face of $\mathcal{E}_{(n+1) \times(n+1)}$ and $V=\bigcap_{X \in F} \operatorname{ker}(X)$. Then, $F$ is a lifting of a face of $\mathcal{E}_{n \times n}$ if and only if there exists a vector $V$ having exactly two nonzero coordinates.

Proof. Necessity is clear. Conversely, suppose that $v \in V$ with $v=(0, \ldots, 0, \alpha, \beta)$. As $v$ is balanced, we deduce that $|\alpha|=|\beta|$, i.e., $\alpha= \pm \beta$. This implies easily that $F$ is a lifting of a face of $\mathcal{E}_{n \times n}$. 


\subsection{The normal cone}

Given a boundary point $x_{0}$ of a convex set $K$, its normal cone $\mathcal{N}\left(K, x_{0}\right)$ is defined by

$$
\mathcal{N}\left(K, x_{0}\right)=\left\{c \in V \mid\langle c, x\rangle \leq\left\langle c, x_{0}\right\rangle \text { for all } x \in K\right\} .
$$

The normal cone $\mathcal{N}\left(\mathcal{E}_{n \times n}, A\right)$ of a matrix $A \in \mathcal{E}_{n \times n}$ will be denoted as $\mathcal{N}(A)$. It can be characterized as follows.

Theorem 2.9 [LP93] We have

$$
\mathcal{N}(A)=\{D-M \mid D \text { is a diagonal matrix, } M \succeq 0,\langle M, A\rangle=0\} .
$$

In fact, we can compute the exact dimension of the normal cone at a correlation matrix $A$, in terms of the rank of $A$.

Theorem 2.10 Let $A \in \mathcal{E}_{n \times n}$ with $q:=\operatorname{dim} \operatorname{ker}(A)$. Then,

$$
\operatorname{dim} \mathcal{N}(A)=\left(\begin{array}{c}
q+1 \\
2
\end{array}\right)+n .
$$

Proof. Let $b_{1}, \ldots, b_{q}$ be linearly independent vectors in $\operatorname{ker}(A)$. Then, the matrices $-\left(b_{i}+b_{j}\right)\left(b_{i}+b_{j}\right)^{t}(1 \leq i \leq j \leq q)$ belong to $\mathcal{N}(A)$. The elementary diagonal matrix $E_{i i}(1 \leq i \leq n)$ is defined as the matrix with all entries 0 but the $(i, i)$-th entry equal 1 . All the $n$ matrices $E_{i i}$ also belong to $\mathcal{N}(A)$. We show that the system $\left\{\left(b_{i}+b_{j}\right)\left(b_{i}+b_{j}\right)^{T} \mid 1 \leq i \leq j \leq q\right\} \cup\left\{E_{i i} \mid 1 \leq i \leq n\right\}$ is linearly independent. For this, let $\lambda_{i j}, \mu_{i}$ be scalars such that

$$
\sum_{1 \leq i \leq j \leq q} \lambda_{i j}\left(b_{i}+b_{j}\right)\left(b_{i}+b_{j}\right)^{t}+\sum_{1 \leq i \leq n} \mu_{i} E_{i i}=0 .
$$

We show that all $\lambda_{i j}$ 's and $\mu_{i}$ 's are equal to 0 . Let $u \in(\operatorname{ker}(A))^{\perp}$. Applying the above relation to $u$, we obtain that $\sum_{1 \leq i \leq n} \mu_{i} E_{i i} u=0$, i.e., $\mu_{i} u_{i}=0$ for all $i=1, \ldots, n$.

Claim 2.11 For all $i \in\{1, \ldots, n\}$, there exists $u \in(\operatorname{ker}(A))^{\perp}$ such that $u_{i} \neq 0$.

Proof. Suppose that $u_{i}=0$ for all $u \in(\operatorname{ker}(A))^{\perp}$. Then, $(\operatorname{ker}(A))^{\perp} \subseteq\left\{u \in \mathbb{R}^{n} \mid\right.$ $\left.u_{i}=0\right\}$. Therefore, $\operatorname{ker}(A) \supseteq\left\{u \in \mathbb{R}^{n} \mid u_{i}=0\right\}^{\perp}$. This implies that the $i$-th unit vector belongs to $\operatorname{ker}(A)$, a contradiction with Theorem 2.4. 
Therefore, $\mu_{i}=0$ for all $i=1, \ldots, n$. Using Lemma 2.1 , we obtain that $\lambda_{i j}=0$ for all $1 \leq i \leq j \leq q$. Hence, we have found a system of $\left(\begin{array}{c}q+1 \\ 2\end{array}\right)+n$ linearly independent members of $\mathcal{N}(A)$. This shows that

$$
\operatorname{dim} \mathcal{N}(A) \geq\left(\begin{array}{c}
q+1 \\
2
\end{array}\right)+n
$$

We now show the converse inequality. Let $\mathcal{B}$ be a system of linearly independent members of $\mathcal{N}(A)$ of maximum cardinality. As all diagonal matrices belong to $\mathcal{N}(A)$, we can suppose without loss of generality that $\mathcal{B}$ is composed of the elementary diagonal matrices $E_{11}, \ldots, E_{n n}$ together with some matrices $-M_{1}, \ldots,-M_{k}$, where each $M_{i}$ is positive semidefinite and satisfies $\left\langle M_{i}, A\right\rangle=0$. By the latter condition, all matrices $M_{i}$ belong to the set $F:=\left\{M \succeq 0 \mid \operatorname{ker}(M) \supseteq(\operatorname{ker}(A))^{\perp}\right\}$. One can check that the set $F$ has dimension $\left(\begin{array}{c}q+1 \\ 2\end{array}\right)$ (see also [HW87]). This implies that $k \leq\left(\begin{array}{c}q+1 \\ 2\end{array}\right)$. Therefore, $\operatorname{dim} \mathcal{N}(A) \leq\left(\begin{array}{c}q+1 \\ 2\end{array}\right)+n$. This concludes the proof.

Note that Theorem 2.10 implies the characterization of the vertices of $\mathcal{E}_{n \times n}$ from Theorem 1.3. Let $A \in \mathcal{E}_{n \times n}$. Suppose that $A$ has rank $r$ and is the Gram matrix of the vectors $v_{1}, \ldots, v_{n} \in \mathbb{R}^{r}$. Set $g:=\operatorname{dim}\left(v_{1} v_{1}^{T}, \ldots, v_{n} v_{n}^{T}\right)$. Then, the dimension of the face $F(A)$ and of the normal cone of $A$ are linked by

$$
\operatorname{dim} F(A)+\operatorname{dim} \mathcal{N}(A)=\left(\begin{array}{c}
n+1 \\
2
\end{array}\right)+n-r(n-r)-g .
$$

(This follows from Theorems 1.1 and 2.10.) This implies

Corollary 2.13

$$
\left(\begin{array}{c}
n+1 \\
2
\end{array}\right)-r(n-r) \leq \operatorname{dim} F(A)+\operatorname{dim} \mathcal{N}(A) \leq\left(\begin{array}{c}
n+1 \\
2
\end{array}\right)-(r-1)(n-r) .
$$

Note that equality holds in the upper bound, for instance, if $A$ is a cut matrix or if $A$ lies in the relative interior of $\mathcal{E}_{n \times n}$.

\section{The dimension of the faces of $\mathcal{E}_{n \times n}$}

We group in this section several results on the faces of the elliptope $\mathcal{E}_{n \times n}$. Using a result of [LT94] recalled in Theorem 1.1 above, we describe all the possible values that can take the dimension of a face of $\mathcal{E}_{n \times n}$; it turns out that the spectrum of feasible dimensions is a union of intervals that ranges from 0 to $\left(\begin{array}{c}n-1 \\ 2\end{array}\right)$. 
Suppose $A \in \mathcal{E}_{n \times n}$ has rank $r$. Then, $A$ is the Gram matrix of a set of vectors $v_{1}, \ldots, v_{n} \in \mathbb{R}^{r}$ of rank $r$; i.e.,

$$
A_{i j}=v_{i}^{T} v_{j} \quad \text { for } 1 \leq i, j \leq n .
$$

A perturbation of $A$ is any symmetric matrix $B$ such that $A \pm t B \in \mathcal{E}_{n \times n}$ for some small $t>0$. Then, the dimension of the face $F(A)$ (the smallest face of $\mathcal{E}_{n \times n}$ containing $A$ ) is defined as the dimension of the space of perturbations of $A$. Let $Z$ denote the $n \times r$ matrix whose columns are $v_{1}, \ldots, v_{n}$; so, $A=Z^{t} Z$. Li and Tam [LT94] show that $B$ is a perturbation of $A$ if and only if

$$
B=Z^{T} R Z
$$

where $R$ belongs to the orthogonal complement of $\left\langle v_{1} v_{1}^{T}, \ldots, v_{n} v_{n}^{T}\right\rangle$ in the space of symmetric $r \times r$ matrices (this latter condition ensures that the diagonal entries of $B$ are equal to 0 ). This implies that the dimension of $F(A)$ can be expressed as in (1.2).

More generally, we have the following result:

ThEOREM 3.2 (i) Let $A \in \mathcal{E}_{n \times n}$ of rank $r$ and let $k$ denote the dimension of $F(A)$. Then, $\left(\begin{array}{c}r+1 \\ 2\end{array}\right)-n \leq k \leq\left(\begin{array}{l}r \\ 2\end{array}\right)$.

(ii) Let $r, k \geq 0$ be integers such that $1 \leq r \leq n$ and $\max \left(0,\left(\begin{array}{c}r+1 \\ 2\end{array}\right)-n\right) \leq k \leq\left(\begin{array}{c}r \\ 2\end{array}\right)$. Then, there exists a matrix $A \in \mathcal{E}_{n \times n}$ of rank $r$ and for which $\operatorname{dim}(F(A))=k$.

Proof. ( $i$ ) follows from the inequalities: $r \leq \operatorname{rank}\left(v_{i} v_{i}^{T} \mid 1 \leq i \leq n\right) \leq n$. (The upper bound is obvious. For the lower bound, observe that the set $\left(v_{1}, \ldots, v_{n}\right)$ has rank $r$ and that if, say, $v_{1}, \ldots, v_{r}$ are linearly independent, then $v_{1} v_{1}^{T}, \ldots, v_{r} v_{r}^{T}$ too are linearly independent, by Lemma 2.1.)

For $(i i)$ we use a construction proposed in [LT94] (also in [GPW90]). Let $e_{1}, \ldots, e_{r} \in$ $\mathbb{R}^{r}$ denote the unit vectors in $\mathbb{R}^{r}$ and set

$$
w_{i j}:=\frac{1}{\sqrt{2}}\left(e_{i}+e_{j}\right) \text { for } 1 \leq i<j \leq r .
$$

One can easily check that the following $\left(\begin{array}{c}r+1 \\ 2\end{array}\right)$ matrices: $\left\{e_{i} e_{i}^{T} \mid 1 \leq i \leq r\right\} \cup$ $\left\{w_{i j} w_{i j}^{T} \mid 1 \leq i<j \leq r\right\}$ are linearly independent.

Suppose first that $n=\left(\begin{array}{c}r+1 \\ 2\end{array}\right)-k$ where $k \leq\left(\begin{array}{c}r \\ 2\end{array}\right)$; hence, $r \leq n \leq\left(\begin{array}{c}r+1 \\ 2\end{array}\right)$. define $A$ as the Gram matrix of the following $n$ vectors: $e_{1}, \ldots, e_{r}$ together with $n-r$ of the vectors $w_{i j}$. By construction, $A$ has rank $r$. Using relation (1.2), one obtains that $\operatorname{dim}(F(A))=\left(\begin{array}{c}r+1 \\ 2\end{array}\right)-n=k$. This shows $(i i)$ in the case when $n=\left(\begin{array}{c}r+1 \\ 2\end{array}\right)-k$. Suppose now that $n>\left(\begin{array}{c}r+1 \\ 2\end{array}\right)-k$. Then, we choose for $A$ a lifting of the matrix defined above; for instance, we can take for $A$ the Gram matrix of the following $n$ vectors: $e_{1}$ (repeated $n-\left(\begin{array}{c}r+1 \\ 2\end{array}\right)+k+1$ times), $e_{2}, \ldots, e_{r}$, together with $\left(\begin{array}{c}r \\ 2\end{array}\right)-k$ of the vectors $w_{i j}$. 
A correlation matrix $X$ is called extreme if $F=\{X\}$ is a 1-dimensional face of $\mathcal{E}_{n \times n}$. Thus, as a special case of Theorem 3.2 we obtain the result of $\mathrm{Li}$ and Tam.

CoRollaRY 3.3 [LT94] Let $r_{\max }$ denote the maximum integer $r$ such that $\left(\begin{array}{c}r+1 \\ 2\end{array}\right) \leq$ n. Then,

(i) $1 \leq \operatorname{rank}(X) \leq r_{\max }$ for every extreme correlation matrix $X \in \mathcal{E}_{n \times n}$.

(ii) For every $r, 1 \leq r \leq r_{\max }$, there is an extreme correlation matrix $X \in \mathcal{E}_{n \times n}$ of rank $r$.

As shown in [LP93], any two cut matrices of $\mathcal{E}_{n \times n}$ form an edge (1-dimensional face) of $\mathcal{E}_{n \times n}$. For $n=3,4$, these are the only edges of $\mathcal{E}_{n \times n}$ (see Section 6). However, for $n \geq 5, \mathcal{E}_{n \times n}$ has edges whose extremities are not cut matrices. A construction for such an edge is given in Example 3.4.

EXAMPLE 3.4 We apply the construction from the proof of Proposition $3.2(i i)$ in the case $n=5, r=3, k=1$. Let $A \in \mathcal{E}_{5 \times 5}$ be the Gram matrix of the vectors $e_{1}=(1,0,0), e_{2}=(0,1,0), e_{3}=(0,0,1), w_{12}=\left(\frac{1}{\sqrt{2}}, \frac{1}{\sqrt{2}}, 0\right)$ and $w_{13}=\left(\frac{1}{\sqrt{2}}, 0, \frac{1}{\sqrt{2}}\right)$, i.e.,

$$
A=\left(\begin{array}{ccccc}
1 & 0 & 0 & \frac{1}{\sqrt{2}} & \frac{1}{\sqrt{2}} \\
0 & 1 & 0 & \frac{1}{\sqrt{2}} & 0 \\
0 & 0 & 1 & 0 & \frac{1}{\sqrt{2}} \\
\frac{1}{\sqrt{2}} & \frac{1}{\sqrt{2}} & 0 & 1 & \frac{1}{2} \\
\frac{1}{\sqrt{2}} & 0 & \frac{1}{\sqrt{2}} & \frac{1}{2} & 1
\end{array}\right) .
$$

Hence, $F(A)$ is an edge of $\mathcal{E}_{5 \times 5}$. In order to describe this edge, we note that $\operatorname{ker}(A)$ is spanned by the vectors

$$
a:=(-1,-1,0, \sqrt{2}, 0), b:=(-1,0,-1,0, \sqrt{2}) .
$$

Then, $X \in \mathcal{E}_{5 \times 5}$ belongs to $F(A)$ if and only if $X a=0$ and $X b=0$. One can check that $X$ must be of the form

$$
X(\alpha):=\left(\begin{array}{ccccc}
1 & 0 & 0 & \frac{1}{\sqrt{2}} & \frac{1}{\sqrt{2}} \\
0 & 1 & \alpha & \frac{1}{\sqrt{2}} & \frac{\alpha}{\sqrt{2}} \\
0 & \alpha & 1 & \frac{\alpha}{\sqrt{2}} & \frac{1}{\sqrt{2}} \\
\frac{1}{\sqrt{2}} & \frac{1}{\sqrt{2}} & \frac{\alpha}{\sqrt{2}} & 1 & \frac{1+\alpha}{2} \\
\frac{1}{\sqrt{2}} & \frac{\alpha}{\sqrt{2}} & \frac{1}{\sqrt{2}} & \frac{1+\alpha}{2} & 1
\end{array}\right),
$$

where $-1 \leq \alpha \leq 1$. Hence the edge $F(A)$ has the matrices $X(-1)$ and $X(1)$ as extremities, where $X(-1)$ and $X(1)$ are of the above form for $\alpha=-1,1$. 
As an application of Theorem 3.2, we can describe the range $\mathcal{D}_{n}$ of the values taken by the dimension of the faces of $\mathcal{E}_{n \times n}$. Namely,

$$
\mathcal{D}_{n}=\left[0,\left(\begin{array}{c}
k_{n} \\
2
\end{array}\right)\right] \cup \bigcup_{k_{n}+1 \leq r \leq n}\left[\left(\begin{array}{c}
r+1 \\
2
\end{array}\right)-n,\left(\begin{array}{l}
r \\
2
\end{array}\right)\right],
$$

where $k_{n}$ is the largest integer such that $\left(\begin{array}{c}k_{n}+1 \\ 2\end{array}\right) \leq n-1$, i.e.,

$$
k_{n}=\left\lfloor\frac{\sqrt{8 n-7}-1}{2}\right\rfloor .
$$

(Given two integers $a, b,[a, b]$ denotes the set of integers $x$ lying between $a$ and $b$.) For instance,

$$
\begin{gathered}
k_{3}=1, \mathcal{D}_{3}=[0,1] \cup\{3\}, \\
k_{4}=2, \mathcal{D}_{4}=[0,3] \cup\{6\}, \\
k_{5}=2, \mathcal{D}_{5}=[0,3] \cup[5,6] \cup\{10\}, \\
k_{6}=2, \mathcal{D}_{6}=[0,6] \cup[9,10] \cup\{15\}, \\
k_{7}=3, \mathcal{D}_{7}=[0,6] \cup[8,10] \cup[14,15] \cup\{21\} .
\end{gathered}
$$

In particular, the largest dimension of a proper face of $\mathcal{E}_{n \times n}$ is $\left(\begin{array}{c}n-1 \\ 2\end{array}\right)$. We give below a direct simple proof of this fact which permits, moreover, to show that every face of $\mathcal{E}_{n \times n}$ of dimension $\left(\begin{array}{c}n-1 \\ 2\end{array}\right)$ is a lifting of $\mathcal{E}_{(n-1) \times(n-1)}$.

Proposition 3.7 Let $F$ be a proper face of $\mathcal{E}_{n \times n}$. Then, $\operatorname{dim}(F) \leq\left(\begin{array}{c}n-1 \\ 2\end{array}\right)$, with equality if and only if $F$ is a lifting of $\mathcal{E}_{(n-1) \times(n-1)}$.

Proof. Let $F$ be a proper face of $\mathcal{E}_{n \times n}$. Then, $F=F_{V}$ for some subspace $V$ of $\mathbb{R}^{n}, V \neq\{0\}$. Let $v \in V, v \neq 0$. We can suppose that $v_{1} \neq 0$. Then, $X v=0$ for all $X \in F$. The equation $X v=0$ can be written as the following system of $n$ equations in the $\left(\begin{array}{l}n \\ 2\end{array}\right)$ variables $x_{i j}(1 \leq i<j \leq n)$ :

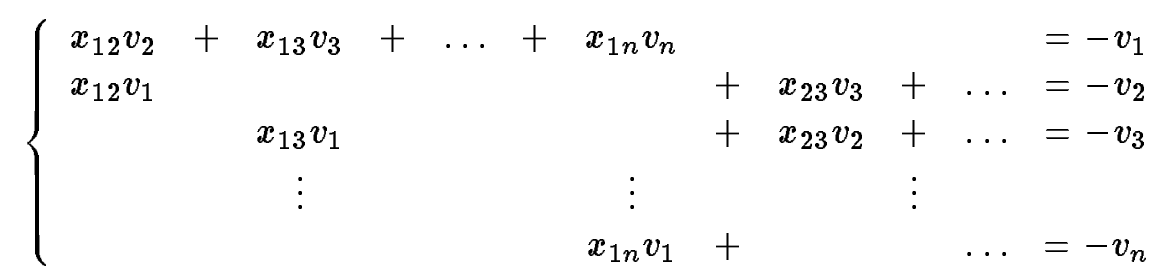

As $v_{1} \neq 0$, the matrix of the system has obviously rank $\geq n-1$. This implies that $\operatorname{dim}(F) \leq\left(\begin{array}{c}n \\ 2\end{array}\right)-(n-1)=\left(\begin{array}{c}n-1 \\ 2\end{array}\right)$. Moreover, the equality $\operatorname{dim}(F)=\left(\begin{array}{c}n-1 \\ 2\end{array}\right)$ holds if and only if the matrix of the system has rank equal to $n-1$. It is not difficult to check that this holds only if $v_{i} v_{j}=0$ for all $2 \leq i<j \leq n$. Hence, we may suppose, for instance, that $v_{3}=v_{4}=\ldots=v_{n}=0$. Hence, $v$ has only two nonzero components. Using Lemma 2.8, we obtain that $F$ is a lifting of a face (of the same 
dimension $\left.\left(\begin{array}{c}n-1 \\ 2\end{array}\right)\right)$ of $\mathcal{E}_{(n-1) \times(n-1)}$. Therefore, $F$ is a lifting of $\mathcal{E}_{(n-1) \times(n-1)}$.

We conclude with an example of a face of the next smaller dimension $\left(\begin{array}{c}n-1 \\ 2\end{array}\right)-1$.

Example 3.8 Consider the face

$$
F:=\left\{X \in \mathcal{E}_{n \times n} \mid X e=0\right\},
$$

where $e$ is the all ones vector. Then, $\operatorname{dim} F=\left(\begin{array}{c}n-1 \\ 2\end{array}\right)-1$. (To see it, one can proceed in the same way as for the proof of Proposition 3.7. Namely, the condition $X e=0$ can be rewritten as the system

$$
\sum_{j=1, \ldots, n, j \neq i} x_{i j}=-1, \text { for all } i=1, \ldots, n .
$$

As the matrix of this sytem has rank $n$, we deduce that $\operatorname{dim} F=\left(\begin{array}{l}n \\ 2\end{array}\right)-n=$ $\left(\begin{array}{c}n-1 \\ 2\end{array}\right)-1$.) Let $X_{0}$ denote the matrix with ones on the diagonal and $-\frac{1}{n-1}$ on the offdiagonal positions. Then, $X_{0}$ belongs to the relative interior of $F$ as $\operatorname{ker}\left(X_{0}\right)=\langle e\rangle$. Hence, $F=F\left(X_{0}\right)$.

Suppose that $n$ is even. Then, $F$ contains the cut matrices $f f^{T}$, for all vectors $f \in\{-1,1\}^{n}$ having exactly $\frac{n}{2}$ entries 1 and $\frac{n}{2}$ entries -1 . Hence, $F$ contains $\frac{1}{2}\left(\begin{array}{c}n \\ n / 2\end{array}\right)$ cut matrices.

Let us look in more detail at the case $n=4$. Then, one can easily check that a matrix $X \in \mathcal{E}_{4 \times 4}$ belongs to the face $F$ if and only if it is of the form

$$
X=\left(\begin{array}{cccc}
1 & x & y & -1-x-y \\
x & 1 & -1-x-y & y \\
y & -1-x-y & 1 & x \\
-1-x-y & y & x & 1
\end{array}\right)
$$

with the conditions: $-1 \leq x, y \leq 1$ and $x+y \leq 0$. Therefore, $F$ is a polyhedral face of $\mathcal{E}_{4 \times 4}$, whose vertices are the three cut matrices $f f^{T}$, for $f=(1,1,-1,-1)$, $(1,-1,-1,1)$, and $(1,-1,1,-1)$.

Finally, note that, for any $n \geq 5$, the face $F$ cannot be a polyhedral face, as its dimension is too large; see Theorem 4.1.

\section{Polyhedral faces of $\mathcal{E}_{n \times n}$}

We consider here the polyhedral faces of the elliptope $\mathcal{E}_{n \times n}$. In particular, we describe the range of their feasible dimensions.

As was mentioned in Proposition 3.7, every face of $\mathcal{E}_{n \times n}$ of dimension $\left(\begin{array}{c}n-1 \\ 2\end{array}\right)$ is isomorphic to $\mathcal{E}_{(n-1) \times(n-1)}$. Hence, $\mathcal{E}_{n \times n}$ has no polyhedral face of dimension 
$\left(\begin{array}{c}n-1 \\ 2\end{array}\right)$. In fact, we can show that the feasible dimensions for polyhedral faces of $\mathcal{E}_{n \times n}$ range from 0 to $k_{n}$, where $k_{n}$ is the largest integer such that $\left(\begin{array}{c}k_{n}+1 \\ 2\end{array}\right) \leq n-1$. We also consider the polyhedral faces of $\mathcal{E}_{n \times n}$ having only cut matrices as vertices, i.e., the faces of $\mathcal{E}_{n \times n}$ that are inherited from the cut polytope. It turns out that such a face is necessarily a simplex. In fact, a simplex face of dimension $k$ can be constructed for any $k \leq k_{n}$.

THEOREM 4.1 Let $F$ be a polyhedral face of $\mathcal{E}_{n \times n}$ of dimension $k-1$. Then, $\left(\begin{array}{l}k \\ 2\end{array}\right) \leq n-1$. Moreover, if all vertices of $F$ are cut matrices, then $F$ is a simplex.

Proof. Let $F_{0} \subset F_{1} \subset \ldots \subset F_{i} \subset F_{i+1} \subset \ldots \subset F_{k-1}:=F$ be a chain of faces of $F$, where $F_{i}$ has dimension $i$ for each $i=0,1, \ldots, k-1$. Using Theorem 2.7, each $F_{i}$ is of the form $F_{V_{i}}=\left\{X \in \mathcal{E}_{n \times n} \mid V_{i} \subseteq \operatorname{ker}(X)\right\}$, where the $V_{i}$ are subspaces of $\mathbb{R}^{n}$ forming a strict chain:

$$
V_{0} \supset V_{1} \supset \ldots \supset V_{i} \supset V_{i+1} \supset \ldots \supset V_{k-1} \text {. }
$$

Then, $\operatorname{dim}\left(V_{k-1}\right) \leq \operatorname{dim}\left(V_{0}\right)-k+1 \leq n-1-k+1=n-k$. Let $X$ be an interior point of $F$ and let $r$ denote the rank of $X$. Then, $r=n-\operatorname{dim}\left(V_{k-1}\right) \geq k$. Using the dimension formula (1.2), we deduce that $k-1=\operatorname{dim}(F) \geq\left(\begin{array}{c}r+1 \\ 2\end{array}\right)-n \geq\left(\begin{array}{c}k+1 \\ 2\end{array}\right)-n$. This implies that $n \geq\left(\begin{array}{l}k \\ 2\end{array}\right)+1$.

Suppose now that all the vertices of $F$ are cut matrices, say, of the form $\left(f_{h} f_{h}^{T}\right.$ $h \in H)$ where $f_{h} \in\{-1,1\}^{n}$ for all $h \in H$. Then, $V_{k-1}=\bigcap_{h \in H} \operatorname{ker}\left(f_{h} f_{h}^{T}\right)=\left\langle f_{h}\right|$ $h \in H\rangle^{\perp}$. Hence, $\operatorname{dim}\left(V_{k-1}\right)=n-\operatorname{dim}\left(\left\langle f_{h} \mid h \in H\right\rangle\right) \leq n-k$, which implies that $\operatorname{dim}\left(\left\langle f_{h} \mid h \in H\right\rangle\right) \geq k$. Let $f_{0}, f_{1}, \ldots, f_{k-1}$ be $k$ linearly independent vectors in the set $\left\{f_{h} \mid h \in H\right\}$. Then, the vertices $f_{i} f_{i}^{T}(i=0,1, \ldots, k-1)$ span affinely the polyhedron $F$. We show that they are the only vertices of $F$. For this, let $X$ be another vertex of $F$. Then, $X=\sum_{0 \leq i \leq k-1} \alpha_{i} f_{i} f_{i}^{T}$ with $\sum_{0 \leq i \leq k-1} \alpha_{i}=1$. We show that each $\alpha_{i}$ is nonnegative. Indeed, let $u \in\left\langle f_{j}\right| j=0,1, \ldots, k-1, j \neq$ $i\rangle^{\perp} \cap\left\langle f_{0}, f_{1}, \ldots, f_{k-1}\right\rangle$ such that $u \neq 0$. Then, $u^{T} X u=\alpha_{i}\left(u^{T} f_{i}\right)^{2} \geq 0$ with $u^{T} f_{i} \neq 0$, yielding $\alpha_{i} \geq 0$. Hence, $X$ is a vertex of $F$ which can be written as a convex combination of other vertices of $F$. This shows that $f_{0} f_{0}^{T}, \ldots, f_{k-1} f_{k-1}^{T}$ are the only vertices of $F$. Therefore, $F$ is a simplex.

We propose below in Proposition 4.7 a construction for polyhedral faces of dimension $k-1$ for each integer $k$ such that $\left(\begin{array}{c}k \\ 2\end{array}\right) \leq n-1$. For this, we state an intermediate result.

We recall the following notation. Given two vectors $x, y \in \mathbb{R}^{n}$, their Hadamard product is the vector $z:=x \circ y \in \mathbb{R}^{n}$ with entries $z_{i}:=x_{i} y_{i}$.

Theorem 4.2 Let $f_{1}, \ldots, f_{k} \in\{-1,1\}^{n}$ and set $e:=(1, \ldots, 1) \in\{-1,1\}^{n}$. Suppose that the following assertions hold: 
(i) The vectors $\left\{f_{1}, \ldots, f_{k}\right\}$ are linearly independent.

(ii) The vectors $\left\{f_{h} \circ f_{h^{\prime}} \mid 1 \leq h<h^{\prime} \leq k\right\} \cup\{e\}$ are linearly independent.

Then, the set $F:=\operatorname{Conv}\left(f_{h} f_{h}^{T} \mid h=1, \ldots, k\right)$ is a face of $\mathcal{E}_{n \times n}$ of dimension $k-1$.

(Here, "Conv" denotes the operation of taking the convex hull.) Note that the face $F$ constructed in the theorem above is a simplex face with cut matrices as vertices.

Proof. Set $X_{0}:=\frac{1}{k}\left(\sum_{1 \leq h \leq k} f_{h} f_{h}^{T}\right)$. Then, $\operatorname{ker}\left(X_{0}\right)=\left\langle f_{1}, \ldots, f_{k}\right\rangle^{\perp}$. Therefore, by $(i), X_{0}$ has rank $k$. Let $F\left(X_{0}\right)$ denote the smallest face of $\mathcal{E}_{n \times n}$ containing $X_{0}$. Clearly, $F\left(X_{0}\right)$ contains $F$. Our goal is to show that $F\left(X_{0}\right)=F$.

Consider the $k \times n$ matrix $M$ whose rows are the vectors $f_{1}, \ldots, f_{k}$. Denote by $v^{1}, \ldots, v^{n} \in \mathbb{R}^{k}$ its columns. Set $w^{i}:=\frac{1}{\sqrt{k}} v^{i}$ for $i=1, \ldots, n$. It is easy to see that $X_{0}$ is equal to the Gram matrix of $w^{1}, \ldots, w^{n}$. Therefore, by the dimension formula (1.2),

$$
\operatorname{dim} F\left(X_{0}\right)=\left(\begin{array}{c}
k+1 \\
2
\end{array}\right)-\operatorname{rank}\left\{w^{1}\left(w^{1}\right)^{T}, \ldots, w^{n}\left(w^{n}\right)^{T}\right\} .
$$

Claim $4.3 \operatorname{rank}\left(w^{1}\left(w^{1}\right)^{T}, \ldots, w^{n}\left(w^{n}\right)^{T}\right) \geq\left(\begin{array}{c}k \\ 2\end{array}\right)+1$.

Proof. By the assumption (ii), the vectors $\left\{f_{h} \circ f_{h^{\prime}} \mid 1 \leq h<h^{\prime} \leq k\right\} \cup\{e\}$ are linearly independent in $\mathbb{R}^{n}$. Let $I$ be a subset of $\{1, \ldots, n\}$ of size $\left(\begin{array}{l}k \\ 2\end{array}\right)+1$ corresponding to the positions of independent coordinates. We show that the set $\left\{w^{i}\left(w^{i}\right)^{T} \mid i \in I\right\}$ is linearly independent. For this suppose that $\sum_{i \in I} \lambda_{i} w^{i}\left(w^{i}\right)^{T}=$ 0 . Note that $w^{i}\left(w^{i}\right)^{T}\left(h, h^{\prime}\right)=\frac{1}{k} f_{h}(i) f_{h^{\prime}}(i)$ which is equal to $\frac{1}{k}\left(f_{h} \circ f_{h^{\prime}}\right)(i)$ if $h \neq h^{\prime}$ and to $\frac{1}{k} e(i)$ if $h=h^{\prime}$. This implies that all $\lambda_{i}$ 's are zero.

As a consequence of the above claim, we deduce that

$$
\operatorname{dim} F\left(X_{0}\right) \leq\left(\begin{array}{c}
k+1 \\
2
\end{array}\right)-\left(\begin{array}{l}
k \\
2
\end{array}\right)-1=k-1 .
$$

On the other hand, $\operatorname{dim} F\left(X_{0}\right) \geq \operatorname{dim}(F)=k-1$. Therefore, $\operatorname{dim} F\left(X_{0}\right)=$ $\operatorname{dim}(F)=k-1$. This implies, in particular, that all the possible perturbations of $X_{0}$ are spanned by $\left\{B_{1}, \ldots, B_{k-1}\right\}$, where

$$
B_{h}:=X_{0}-f_{h} f_{h}^{T} .
$$

Now, we can show that $F\left(X_{0}\right)=F$. Let $X \in F\left(X_{0}\right)$. Hence, $X=X_{0}+B$ where $B$ is a perturbation of $X_{0}$. By the above observation, $B=\sum_{1 \leq h \leq k-1} \lambda_{h}\left(X_{0}-f_{h} f_{h}^{T}\right)$ for some scalars $\lambda_{h}$. Therefore, setting $\lambda:=\sum_{1 \leq h \leq k-1} \lambda_{h}$, we obtain that

$$
X=\sum_{1 \leq h \leq k-1}\left(-\lambda_{h}+\frac{1}{k}(1+\lambda)\right) f_{h} f_{h}^{T}+\frac{1}{k}(1+\lambda) f_{k} f_{k}^{T}
$$


The sum of coefficients is equal to 1 . This implies that $X$ belongs to the affine hull of $\left\{f_{1} f_{1}^{T}, \ldots, f_{k} f_{k}^{T}\right\}$. Now, using an argument similar to the one used in the proof of Theorem 4.1, we can conclude that $X \in F$. (Indeed, if $X=\sum_{1 \leq h \leq k} \mu_{h} f_{h} f_{h}^{T}$ with $\sum_{1 \leq h \leq k} \mu_{h}=1$, then $\mu_{h} \geq 0$ for all $h$. To see it, take a nonzero vector $u$ in the intersection of the spaces $\left\langle f_{1}, \ldots, f_{k}\right\rangle$ and $\left\langle f_{1}, \ldots, f_{k-1}\right\rangle^{\perp}$. Then, $u^{T} X u=$ $\mu_{k}\left(u^{T} f_{k}\right)^{2} \geq 0$ implying that $\mu_{k} \geq 0$. The same argument shows that all $\mu_{h}$ 's are nonnegative.)

RemarK 4.4 We can suppose without loss of generality in Theorem 4.2 that the vectors $f_{1}, \ldots, f_{k}$ have a common entry equal to 1 , say, $f_{h}(n)=1$ for $h=1, \ldots, k$. Set $S_{h}:=\left\{i \mid f_{h}(i)=1\right\}$ for $h=1, \ldots, k$. It is easy to check that the assumption (ii) of Theorem 4.2 can be reformulated as:

(iii) The $\left(\begin{array}{c}k \\ 2\end{array}\right)$ vectors $\chi^{S_{h} \Delta S_{h^{\prime}}}\left(1 \leq h<h^{\prime} \leq k\right)$ are linearly independent. (Here, $\chi^{A}$ denotes the 0,1 -incidence vector of the set $A$.)

Let us recall that the sets $S_{1}, \ldots, S_{k} \subseteq V:=\{1, \ldots, n\}$ are said to be in general position if each of the intersection sets $C(H):=\bigcap_{h \in H} S_{h} \cap \bigcap_{h \notin H}\left(V \backslash S_{h}\right)$ is nonempty, for every $H \subseteq\{1, \ldots, k\}$.

We say that the vectors $f_{1}, \ldots, f_{k} \in\{-1,1\}^{n}$ are in general position if the sets $S_{h}:=\left\{i \mid f_{h}(i)=1\right\}$ are in general position.

Corollary 4.5 Let $f_{1}, \ldots, f_{k} \in\{-1,1\}^{n}$ be in general position. Then, the set $\operatorname{Conv}\left(f_{1} f_{1}^{T}, \ldots, f_{k} f_{k}^{T}\right)$ is a face of $\mathcal{E}_{n \times n}$.

Proof. By Theorem 4.2 and Remark 4.4, it suffices to verify that the conditions (i) and (iii) hold, which can be easily done.

EXAMPLE 4.6 Let $n=4, f_{1}=(1,-1,-1,-1), f_{2}=(1,-1,1,1), f_{3}=(1,1,-1,1)$. The sets $S_{1}:=\{1\}, S_{2}:=\{1,3,4\}, S_{3}:=\{1,2,4\}$ are not in general position but satisfy nevertheless the assumption (iii). Also $(i)$ holds. Hence, the set $\operatorname{Conv}\left(f_{1} f_{1}^{T}, f_{2} f_{2}^{T}, f_{3} f_{3}^{T}\right)$ is a polyhedral face of $\mathcal{E}_{4 \times 4}$ of dimension 2 . Note that this face falls into the category of the so-called elliptic faces of $\mathcal{E}_{4 \times 4}$ (see Section 6). Also, $F=F_{V}$ where $V=\left\langle f_{1}, f_{2}, f_{3}\right\rangle^{\perp}=\langle(1,1,1,-1)\rangle$.

Proposition 4.7 For each integer $k$ such that $\left(\begin{array}{c}k \\ 2\end{array}\right)+1 \leq n$, the elliptope $\mathcal{E}_{n \times n}$ has a polyhedral face of dimension $k-1$ (which is a simplex with cut matrices as vertices). 
Proof. It is enough to show it for $n=\left(\begin{array}{c}k \\ 2\end{array}\right)+1$ (for larger values of $n$, apply lifting). Let $G$ denote the graph with node set $\{1, \ldots, k, k+1\}$, obtained from the complete graph $K_{k}$ on $\{1, \ldots, k\}$ by adding an edge $e$, say $e=(1, k+1)$. We consider the edge set of $G$ as our groundset of $n$ elements. For $h=1, \ldots, k$, let $S_{h}$ denote the set of edges in the star of the node $h$ plus the edge $e$, i.e., $S_{h}$ consists of the edges $(h, i)(i \in\{1, \ldots, k\} \backslash\{h\})$ together with the edge $e$. Let $f_{h}$ denote the \pm 1 -incidence vector of $S_{h}$. Then, $\operatorname{Conv}\left(f_{1} f_{1}^{T}, \ldots, f_{k} f_{k}^{T}\right)$ is a face of $\mathcal{E}_{n \times n}$ (as the assumptions $(i),(i i i)$ can be easily checked to hold).

As an application of Theorem 4.1 and Proposition 4.7, we obtain that the largest dimension of a polyhedral face of $\mathcal{E}_{n \times n}$ is equal to $k_{n}$, where $k_{n}$ is defined by (3.6), i.e., $k_{n}$ is the largest integer such that $\left(\begin{array}{c}k_{n}+1 \\ 2\end{array}\right) \leq n-1$.

Corollary 4.8 The maximum dimension of a polyhedral face of the elliptope $\mathcal{E}_{n \times n}$ is

$$
\left\lfloor\frac{\sqrt{8 n-7}-1}{2}\right\rfloor \text {. }
$$

REMARK 4.9 It was shown in [DLP92] that, if the vectors $f_{1}, \ldots, f_{k}$ are in general position, then the set $F:=\operatorname{Conv}\left(f_{1} f_{1}^{T}, \ldots, f_{k} f_{k}^{T}\right)$ is a face of the metric polytope and, thus, of the cut polytope $\mathrm{CUT}_{n \times n}$. We recall that the the metric polytope is defined by the set of linear inequalities

$$
\begin{array}{lll}
\operatorname{MET}_{n \times n}:=\left\{X \in \mathrm{SYM}_{n \times n} \mid\right. & X_{i i}=1 & \text { for } i=1, \ldots, n \\
& X_{i j}-X_{i k}-X_{j k} \geq-1 & \text { for } 1 \leq i, j, k \leq n \\
& \left.X_{i j}+X_{i k}+X_{j k} \geq-1 \quad \text { for } 1 \leq i, j, k \leq n\right\}
\end{array}
$$

(Thus, the metric polytope is a linear relaxation of the cut polytope; see [LPR94] for more details.) Corollary 4.5 shows that the set $F$ is also a face of the elliptope $\mathcal{E}_{n \times n}$.

\section{Optimization aspects}

Let us consider the optimization problem

$$
\begin{gathered}
\min \langle C, X\rangle \\
X \in \mathcal{E}_{n \times n}
\end{gathered}
$$


where $C$ is a symmetric $n \times n$ matrix. Recall that

$$
\langle C, X\rangle=\operatorname{Tr}(C X)=\sum_{i, j=1, \ldots, n} c_{i j} x_{i j}
$$

This problem is of interest, because it is related to the max-cut problem. To be more precise, the problem

$$
\begin{array}{cc}
\max \frac{1}{2} \sum_{1 \leq i<j \leq n} c_{i j}\left(1-x_{i j}\right)= & \frac{1}{4}\langle C, J\rangle-\frac{1}{4} \min \langle C, X\rangle \\
X \in \mathcal{E}_{n \times n} & X \in \mathcal{E}_{n \times n}
\end{array}
$$

provides a good approximation of the max-cut problem:

$$
\begin{array}{cl}
\frac{1}{2} \max & \sum_{1 \leq i<j \leq n} c_{i j}\left(1-a_{i} a_{j}\right) \\
& a \in\{-1,1\}^{n}
\end{array}
$$

(For various results concerning the approximation of (5.3) by (5.2) we refer to the following papers: worst case bound of the approximation [GW94], asymptotic optimality of the approximation [DP93a], complexity and further aspects [DP93b, LP93].)

Let $F_{C}$ denote the set of optimum solutions to the problem (5.2), i.e.,

$$
F_{C}=\left\{A \in \mathcal{E}_{n \times n} \mid\langle C, A\rangle \leq\langle C, X\rangle \text { for all } X \in \mathcal{E}_{n \times n}\right\} \text {. }
$$

The set $F_{C}$ is exposed. Let us recall that a set $F$ is called an exposed set of a convex set $K$ if $F=K \cap H$ for some supporting hyperplane $H$ for $K$. Clearly, each exposed set is a face of $K$. For a general convex set $K$, the converse is not true. However, for the elliptope $\mathcal{E}_{n \times n}$ both notions coincide.

Lemma 5.4 [LP93] Every face of $\mathcal{E}_{n \times n}$ is exposed.

If $F_{C}$ contains a rank one matrix, then (5.2) provides an exact solution of the max cut problem. Hence we are interested in finding low-rank matrices in $F_{C}$, since they (intuitively) provide a tighter approximation of the max-cut.

QUESTION 5.5 Given a face $F$ of $\mathcal{E}_{n \times n}$, what is the minimum rank of a matrix $X \in F$ ?

Since there exist extreme correlation matrices of any rank $r$ up to the bound $r_{\max }$ given in Corollary 3.3, we cannot ensure, in general, the existence of matrices with rank smaller that $r_{\max } \approx \sqrt{2 n}$. However, we are able to establish the existence of a low rank matrix under some additional constraints.

Lemma 5.6 For every balanced vector $c \in \mathbb{R}^{n}$, there is a matrix $X \in \mathcal{E}_{n \times n}$ such that $c \in \operatorname{ker}(X)$ and $\operatorname{rank}(X) \leq 2$. 
Proof. Without loss of generality, we may assume that $c_{1} \geq c_{2} \geq \ldots c_{n} \geq 0$. Let $i_{0}$ be such that

$$
\sum_{j<i_{0}} c_{j} \leq \sum_{j \geq i_{0}} c_{j} \quad \text { and } \quad \sum_{j \leq i_{0}} c_{j} \geq \sum_{j>i_{0}} c_{j}
$$

Set $\bar{c}_{1}:=\sum_{j<i_{0}} c_{j}, \bar{c}_{2}=c_{i_{0}}$ and $\bar{c}_{3}:=\sum_{j>i_{0}} c_{j}$. Then, it easily follows that $\bar{c}=\left(\bar{c}_{1}, \bar{c}_{2}, \bar{c}_{3}\right)$ is balanced, since $\bar{c}_{1}+\bar{c}_{2} \geq \bar{c}_{3}, \bar{c}_{1} \leq \bar{c}_{2}+\bar{c}_{3}$ by the choice of $i_{0}$, and $\bar{c}_{2}=c_{i_{0}} \leq c_{1} \leq \bar{c}_{1}+\bar{c}_{3}$ by the nonnegativity of $c$. Since $\bar{c} \in \mathbb{R}^{3}$ is balanced, there exists a matrix $\bar{X} \in \mathcal{E}_{3 \times 3}$ with $\bar{c} \in \operatorname{ker}(\bar{X})$ (by Theorem 2.4). Set

$$
\bar{X}=\left(\begin{array}{ccc}
1 & a & b \\
a & 1 & c \\
b & c & 1
\end{array}\right) \text { and } X=\left(\begin{array}{c|c|c} 
& a & \\
J & \vdots & b J \\
& a & \\
\hline a \ldots a & 1 & c \ldots c \\
\hline & c & \\
b J & \vdots & J
\end{array}\right)
$$

where we have specified the $i_{0}$-th row and $i_{0}$-th column in $X$ and $J$ denotes the all ones matrix (of appropriate sizes). Then, $\operatorname{rank}(X)=\operatorname{rank}(\bar{X}) \leq 2$ and $c \in \operatorname{ker}(X)$.

THEOREM 5.7 If a face $F$ of $\mathcal{E}_{n \times n}$ contains a matrix of rank $n-1$, then it also contains a matrix of rank at most two.

Proof. The statement holds trivially if $F=\mathcal{E}_{n \times n}$. Suppose now that $F=F(A)$ where $A$ has rank $n-1$. By Lemma 5.6, there exists $B \in \mathcal{E}_{n \times n}$ of rank $\leq 2$ such that $\operatorname{ker}(A) \subseteq \operatorname{ker}(B)$, i.e., $B \in F$.

Note that, under the assumption of Theorem $5.7, \operatorname{dim}(F)=\left(\begin{array}{c}n-1 \\ 2\end{array}\right)-1,\left(\begin{array}{c}n-1 \\ 2\end{array}\right)$, or $\left(\begin{array}{l}n \\ 2\end{array}\right)$.

EXAMPLE 5.8 The construction from the proof of Proposition 3.2 (which was already applied in Example 3.4) for the parameters: $n=9, r=4, k=1$ provides a matrix $A$ of rank 4 whose face is an edge. One can determine the extremities of this edge (as was done in Example 3.4) and check that their ranks are equal to 3. So this gives a face containing only matrices of ranks 3 and 4 . 
Also the dual problem of (5.2) is of interest. The dual problem reads:

$$
\begin{array}{ll}
\frac{n}{4} \min & \lambda_{\max }\left(L_{C}+\operatorname{diag}(u)\right) \\
& u_{1}+\ldots+u_{n}=0
\end{array}
$$

We recall that $L_{C}$ denotes the Laplacian matrix; it is the $n \times n$ symmetric matrix with $(i, i)$-th diagonal entry $\sum_{j=1, \ldots, n, j \neq i} c_{i j}$ and with $(i, j)$-entry $-c_{i j}$ for $i \neq j$. (Note that $L_{C}$ does not depend on the diagonal entries of $C$.) Let $u$ denote the optimum vector for the program (5.9), set $\lambda:=\lambda_{\max }\left(L_{C}+\operatorname{diag}(u)\right)$ and let $V_{\text {eig }}$ denote the eigenspace corresponding to this eigenvalue for the matrix $L_{C}+\operatorname{diag}(u)$. It has been shown that strong duality holds, i.e., that both programs (5.2) and (5.9) have the same optimum solutions. Since the maximum eigenvalue in the optimum is typically multiple (unless the corresponding eigenvector is a \pm 1 vector, in which case (5.1) provides an exact solution of the max-cut), the following question was asked in [DP93b], and in a more general setting also in [Ov88].

QUESTION 5.10 What is the possible dimension of the space $V_{\text {eig }}$ ?

The next result establishes a link between the eigenspace $V_{e i g}$ and the face $F_{C}$ and implies a lower bound for the dimension of $V_{e i g}$.

Proposition 5.11 We have

$$
F_{C}=\left\{X \in \mathcal{E}_{n \times n} \mid \operatorname{ker}(X) \supseteq\left(V_{e i g}\right)^{\perp}\right\} .
$$

Proof. Set $M:=\lambda I-L_{C}-\operatorname{diag}(u)$. By construction, $M$ is a positive semidefinite matrix and its kernel is $\operatorname{ker}(M)=V_{\text {eig }}$. For $X \in \mathcal{E}_{n \times n}$, we have

$$
\left\langle L_{C}, X\right\rangle=\sum_{i, j} L_{C}(i, j) x_{i j}=2 \sum_{1 \leq i<j \leq n} c_{i j}\left(1-x_{i j}\right),
$$

which implies

$$
\begin{aligned}
\langle M, X\rangle & =\langle\lambda I, X\rangle-\left\langle L_{C}, X\right\rangle-\langle\operatorname{diag}(u), X\rangle \\
& =\lambda n-4\left(\sum_{1 \leq i<j \leq n} \frac{c_{i j}}{2}\left(1-x_{i j}\right)\right) \geq 0 .
\end{aligned}
$$

Therefore, we see that $\langle M, X\rangle=0$ if and only if $X$ is an optimum solution to the program (5.2), i.e., if $X \in F_{C}$. Suppose $M=\sum_{1 \leq i \leq k} u_{i} u_{i}^{T}$, where $u_{1}, \ldots, u_{k}$ span the space $(\operatorname{ker}(M))^{\perp}$. Then, $\langle M, X\rangle=0$ holds if and only if $X u_{i}=0$ for all $i=1, \ldots, k$, i.e., if $(\operatorname{ker}(M))^{\perp} \subseteq \operatorname{ker}(X)$. This shows the result.

Corollary 5.12 For every matrix $X \in F_{C}, \operatorname{rank}(X) \leq \operatorname{dim}\left(V_{\text {eig }}\right)$. 
An alternative proof of Corollary 5.12 can be given as follows. Since $X \succeq 0$, we have $X=Z^{T} Z$ for a matrix $Z$ of the same rank as $X$. It can be checked that the rows of $Z$ are eigenvectors from the space $V_{\text {eig }}$. Hence $\operatorname{rank}(X)=\operatorname{rank}(Z) \leq$ $\operatorname{dim}\left(V_{\text {eig }}\right)$.

Example 5.13 Consider the cost matrix $C:=J$. Then, the Laplacian matrix is $L_{C}=n I-J$. Then, $\min _{u^{T} e=0} \lambda_{\max }\left(L_{C}+\operatorname{diag}(u)\right)$ is attained for $u=0$ (by symmetry, see [DP93a]) and is equal to $\lambda_{\max }\left(L_{C}\right)=n$. The optimized eigenspace is $V_{\text {eig }}=\left\{x \in \mathbb{R}^{n} \mid \sum_{1 \leq i \leq n} x_{i}=0\right\}$, with dimension $n-1$. Hence, by Proposition 5.11 , the face $F_{C}$ is $\left\{\bar{X} \in \mathcal{E}_{n \times n} \mid X e=0\right\}$. Note that it coincides with the face considered in Example 3.8. In particular, $\left(V_{\text {eig }}\right)^{\perp}=\operatorname{ker}(X)$ for every matrix $X$ lying in the relative interior of $F_{C}$.

By Corollary 5.12, $\operatorname{rank}(X) \leq \operatorname{dim}\left(V_{\text {eig }}\right)$ for each matrix $X$ lying in the relative interior of $F_{C}$. In the above example, we have equality: $\operatorname{rank}(X)=\operatorname{dim}\left(V_{e i g}\right)$. However, as shown in the following example, strict inequality may hold and, in fact, the gap can be made as large as possible.

Example 5.14 Consider the cost matrix $C$ defined by $c_{1 j}=1$ for all $j=2, \ldots, n$ and $c_{i j}=\frac{1}{n-1}$ for all $2 \leq i<j \leq n$. Then, the Laplacian matrix has the form

$$
L_{C}=\left(\begin{array}{ccccc}
n-1 & -1 & \ldots & \cdots & -1 \\
-1 & \frac{2 n-3}{n-1} & & & \\
\vdots & & \ddots & -\frac{1}{n-1} & \\
\vdots & & -\frac{1}{n-1} & \ddots & \\
-1 & & & & \frac{2 n-3}{n-1}
\end{array}\right)
$$

Then, the optimizing vector $u$ for $\min _{u^{T} e=0} \lambda_{\max }\left(L_{C}+\operatorname{diag}(u)\right)$ satisfies $u_{2}=\ldots=$ $u_{n}$ (by symmetry, see [DP93b]). Using this fact, it is not difficult to check that the optimum vector $u$ is $(-(n-1) a, a, \ldots, a)$ for $a=\frac{2(n-2)}{n}$. Then, the optimum value of $\lambda_{\max }\left(L_{C}+\operatorname{diag}(u)\right)=\frac{4(n-1)}{n}$. Moreover, the optimized eigenspace is $V_{e i g}=\left\{x \in \mathbb{R}^{n} \mid(n-1) x_{1}+\sum_{2 \leq i \leq n} x_{i}=0\right\}$, with dimension $n-1$. Hence, $\left(V_{e i g}\right)^{\perp}$ is spanned by the vector $v=(n-1,1, \ldots, 1)$. Therefore, by Proposition 5.11 , the face $F_{C}$ is given by

$$
F_{C}=\left\{X \in \mathcal{E}_{n \times n} \mid X v=0\right\} .
$$

As $v$ is not strictly balanced, we know from Theorem 2.6 that there cannot exist a matrix in $F_{C}$ whose kernel is spanned by $v$. In fact, one can check that the only 
matrix of $\mathcal{E}_{n \times n}$ satisfying $X v=0$ is the cut matrix

$$
X_{0}:=\left(\begin{array}{c|c}
1 & -1 \ldots-1 \\
\hline-1 & \\
\vdots & J \\
-1 &
\end{array}\right)
$$

Hence, the rank of $X_{0}$ is 1 while the dimension of $\left(V_{e i g}\right)^{\perp}$ is $n-1$, which is the largest possible gap.

From the characterization of the normal cone (of Theorem 2.9) can be derived the following alternative description of the face $F_{C}$. Indeed,

$$
\begin{aligned}
A \in F_{C} \Longleftrightarrow & -C \in \mathcal{N}(A) \\
\Longleftrightarrow & \exists D \text { diagonal matrix such that } \\
& C+D \succeq 0, \operatorname{ker}(C+D) \supseteq(\operatorname{ker} A)^{\perp} .
\end{aligned}
$$

Therefore,

$$
F_{C}=\left\{X \in \mathcal{E}_{n \times n} \mid \operatorname{ker} X \supseteq(\operatorname{ker}(C+D))^{\perp} \text { for some diagonal matrix } D\right\} .
$$

An interesting question is whether it is possible, given a cost matrix $C$, to find an element of $F_{C}$ (of smallest possible rank) not using some classical optimization algorithm, but using rather some algebraic techniques based, for instance, on the above description of $F_{C}$.

\section{The elliptope $\mathcal{E}_{4 \times 4}$}

In this section, we give a description of the faces of the set $\mathcal{E}_{4 \times 4}$ of $4 \times 4$ correlation matrices. This question was raised by W. Barrett (private communication, 1994). Note that $\mathcal{E}_{4 \times 4}$ is a convex set of dimension 6 .

THEOREM 6.1 Let $F$ be a proper face of $\mathcal{E}_{4 \times 4}$. Then, one of the following holds.

(i) $\operatorname{dim}(F)=0$, i.e., $F$ consists of a unique matrix (which is an extreme element of $\left.\mathcal{E}_{4 \times 4}\right)$.

(ii) $F$ is an edge joining two cut matrices, so $\operatorname{dim}(F)=1$. There are $\left(\begin{array}{l}8 \\ 2\end{array}\right)=28$ such faces.

(iii) $F$ is an elliptic face, $\operatorname{dim}(F)=2$.

(iv) $F$ is isomorphic to $\mathcal{E}_{3 \times 3}$ (more precisely, $F$ is a lifting of $\mathcal{E}_{3 \times 3}$ ), so $\operatorname{dim}(F)=3$. There are 8 such faces. 
Hence, we find again that the range of feasible dimensions for the faces of $\mathcal{E}_{4 \times 4}$ is $[0,3] \cup\{6\}$; recall (3.5). According to Corollary 4.5, the highest dimension of a polyhedral face of $\mathcal{E}_{4 \times 4}$ is 2 ; recall the construction of such a face from Example 4.6. The elliptope $\mathcal{E}_{4 \times 4}$ has also nonpolyhedral faces of dimension 2; see Examples 6.5 and 6.6 below.

We call a face of dimension 2 of $\mathcal{E}_{4 \times 4}$ an elliptic face because, as will be seen in the proof, it is described by a set of inequalities $f(x, y) \geq 0$, where $f$ is a polynomial of degree less than or equal to 2 in the variables $x, y$.

Proof of Theorem 6.1. Let $F$ be a face of $\mathcal{E}_{4 \times 4}$. Suppose first that $F$ arises as a lifting of a face $G$ of $\mathcal{E}_{3 \times 3}$. We use the description of the faces of $\mathcal{E}_{3 \times 3}$ given in Proposition 2.10 from [LP93]. Either $G=\mathcal{E}_{3 \times 3}$ in which case $F$ is one of the faces from Theorem $6.1(i v)$. Either $G$ is an edge between two cut matrices in which case $F$ is one of the faces from case (ii). It may be also that $G$ is reduced to a single element in which case $F$ is also reduced to a single element; hence we are in the situation $(i)$. From now on we suppose that $F$ is not a lifting of a face of $\mathcal{E}_{3 \times 3}$. Set $V=\bigcap_{X \in F} \operatorname{ker}(X)$. By Lemma 2.8, every vector of $V$ has at least three nonzero components. We distinguish several cases depending on the dimension of $V$.

Case 1: $\operatorname{dim}(V)=1$. Let $v \in V, v \neq 0$. We can suppose that $v=(1, a, b, c)$, where at least two of $a, b, c$ are nonzero. We can suppose that $a, b \neq 0$. Let

$$
X=\left(\begin{array}{cccc}
1 & x & y & z \\
x & 1 & x^{\prime} & y^{\prime} \\
y & x^{\prime} & 1 & z^{\prime} \\
z & y^{\prime} & z^{\prime} & 1
\end{array}\right)
$$

be a matrix of $F$. The condition: $X v=0$ can be rewritten as the system

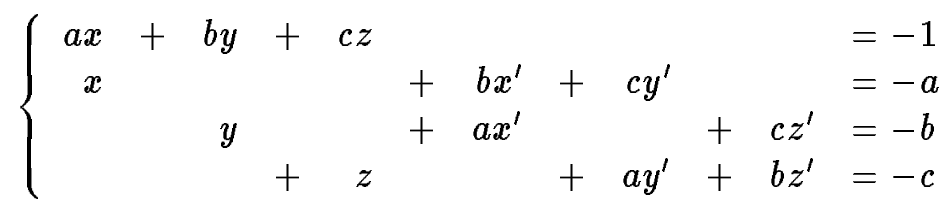

in the variables $x, y, z, x^{\prime}, y^{\prime}, z^{\prime}$. As $a, b \neq 0$, the variables $x, y, z, x^{\prime}$ can be uniquely expressed in terms of $a, b, c, y^{\prime}, z^{\prime}$. Namely,

$$
\left\{\begin{array}{l}
x=\frac{1}{2 a}\left(-1-a^{2}+b^{2}+c^{2}+2 b c z^{\prime}\right) \\
y=\frac{1}{2 b}\left(-1+a^{2}-b^{2}+c^{2}+2 a c y^{\prime}\right) \\
z=-a y^{\prime}-b z^{\prime}-c \\
x^{\prime}=\frac{1}{2 a b}\left(1-a^{2}-b^{2}-c^{2}-2 a c y^{\prime}-2 b c z^{\prime}\right) .
\end{array}\right.
$$

The condition: $X \succeq 0$ can be expressed by asking that all $2 \times 2$ and $3 \times 3$ principal subdeterminants of $X$ be nonnegative, i.e., 


$$
\left\{\begin{array}{l}
-1 \leq x, y, z, x^{\prime}, y^{\prime}, z^{\prime} \leq 1 \\
1-x^{2}-y^{2}-\left(x^{\prime}\right)^{2}+2 x y x^{\prime} \geq 0 \\
1-x^{2}-z^{2}-\left(y^{\prime}\right)^{2}+2 x z y^{\prime} \geq 0 \\
1-y^{2}-z^{2}-\left(z^{\prime}\right)^{2}+2 y z z^{\prime} \geq 0 \\
1-\left(x^{\prime}\right)^{2}-\left(y^{\prime}\right)^{2}-\left(z^{\prime}\right)^{2}+2 x^{\prime} y^{\prime} z^{\prime} \geq 0 .
\end{array}\right.
$$

Hence, $F$ is a face of dimension 2, which is determined by the systems (6.3) and (6.4). So, the boundary of $F$ is described by polynomial equations in the variables $y^{\prime}, z^{\prime}$ of degree less than or equal to 2 . Therefore, $F$ is an elliptic face as in Theorem $6.1($ iii) .

Case 2: $\operatorname{dim}(V)=2$. Let $X \in F$ that is not a cut matrix. Then, $\operatorname{ker}(X)=V$ (else, $\operatorname{ker}(X)$ has dimension 3 which implies that $X$ is a cut matrix). This shows that, if $F$ is not reduced to a single element, then its relative boundary consists only of cut matrices and, thus, $F$ is an edge between two cut matrices. However, we have already ruled out this possibility (as we assume that $F$ is not a lifting of a face of $\mathcal{E}_{3 \times 3}$ ). Therefore, $F$ is reduced to a single element, i.e., we are in the situation of Theorem $6.1(i)$.

Case 3: $\operatorname{dim}(V)=3$. Then, $F$ is reduced to one element which is a cut matrix. So we are in the situation of Theorem $6.1(i)$.

We recall Example 4.6, where was described a polyhedral elliptic face of $\mathcal{E}_{4 \times 4}$, namely, the face $\left\{X \in \mathcal{E}_{4 \times 4} \mid X v=0\right\}$ where $v=(1,1,1,-1)^{T}$. Also in Example 3.8 was described the polyhedral face of $\mathcal{E}_{4 \times 4}$ corresponding to the vector $v=(1,1,1,1)^{T}$.

We now present two examples of nonpolyhedral elliptic faces of $\mathcal{E}_{4 \times 4}$. They are of the form $F=\left\{X \in \mathcal{E}_{4 \times 4} \mid X v=0\right\}$ where $v \in \mathbb{R}^{4}$ is a balanced vector.

Example 6.5 Take $v=(1,1,1,0)^{T}$. Then, $F$ consists of the matrices

$$
\left(\begin{array}{rrcc}
1 & -\frac{1}{2} & -\frac{1}{2} & x \\
-\frac{1}{2} & 1 & -\frac{1}{2} & y \\
-\frac{1}{2} & -\frac{1}{2} & 1 & -x-y \\
x & y & -x-y & 1
\end{array}\right)
$$

where $x, y$ satisfy the condition: $x^{2}+x y+y^{2} \leq \frac{3}{4}$. Hence, $F$ has really the shape of an ellipse.

Example 6.6 Let $v=(1,1,2,1)^{T}$. Then, $F$ consists of the matrices (6.2) satisfying (6.3) and (6.4), where (6.3) reads

$$
x=\frac{1}{2}\left(3+4 z^{\prime}\right), y=\frac{1}{4}\left(-3+2 y^{\prime}\right), z=\frac{1}{4}\left(-5-2 y^{\prime}-4 z^{\prime}\right), x^{\prime}=-1-y^{\prime}-2 z^{\prime} .
$$




\section{References}

[BJT93] W. Barrett, C.R. Johnson, and P. Tarazaga. The real positive definite completion problem for a simple cycle. Linear Algebra and its Applications, 192:3-31, 1993.

[CM79] J.P.R. Christensen and J. Vesterstrøm. A note on extreme positive definite matrices. Mathematische Annalen, 244:65-68, 1979.

[DP93a] C. Delorme and S. Poljak. Laplacian eigenvalues and the maximum cut problem. Mathematical Programming, 62:557-574, 1993.

[DP93b] C. Delorme and S. Poljak. Combinatorial properties and the complexity of an eigenvalue approximation of the max-cut problem, European Journal of Combinatorics, 14:313-333, 1993.

[DLP92] M. Deza, M. Laurent, and S. Poljak. The cut cone III: on the role of triangle facets. Graphs and Combinatorics, 8:125-142, 1992. (Updated version in Graphs and Combinatorics, 9:135-152, 1993.)

[DG81] H. Dym and I. Gohberg. Extensions of band matrices with band inverses. Linear Algebra and its Applications, 36:1-24, 1981.

[GW94] Michel X. Goemans and David P. Williamson. Improved approximation algorithms for maximum cut and satisfiability problems using semidefinite programming. In Proceedings of the 26th annual symposium on Theory of Computing, Montréal, pages 422-431, 1994.

[GJSW84] R. Grone, C.R. Johnson, E.M. Sá, and H. Wolkowicz. Positive definite completions of partial hermitian matrices. Linear Algebra and its Applications, 58:109-124, 1984.

[GPW90] R. Grone, S. Pierce, and W. Watkins. Extremal correlation matrices. Linear Algebra and its Applications, 134:63-70, 1990.

[HW87] R.D. Hill and S.R. Waters. On the cone of positive semidefinite matrices. Linear Algebra and its Applications, 90:81-88, 1987.

[La94] M. Laurent. The real positive semidefinite completion problem for series-parallel graphs. Preprint, 1994. 
[LP92] M. Laurent and S. Poljak. One-third-integrality in the metric polytope. Report BS-R9209, Centrum voor Wiskunde en Informatica (Amsterdam), 1992. (To appear in Mathematical Programming.)

[LP93] M. Laurent and S. Poljak. On a positive semidefinite relaxation of the cut polytope. Rapport LIENS-93-27, Ecole Normale Supérieure (Paris), 1993. (To appear in Linear Algebra and its Applications.)

[LPR94] M. Laurent, S. Poljak, and F. Rendl. Connections between semidefinite relaxations of the max-cut and stable-set problems. Preprint, 1994.

[LRT76] G.S. Leuker, D.J. Rose, and R.E. Tarjan. Algorithmic aspects of vertex eliminations on graphs. SIAM Journal on Computation, 5(2):266-283, 1976 .

[LT94] Chi-Kwong Li and Bit-Shun Tam. A note on extreme correlation matrices. SIAM Journal on Matrix Analysis and its Appplications, 15(3):903-908, 1994.

[Loe80] R. Loewy. Extreme points of a convex subset of the cone of positive semidefinite matrices. Mathematische Annalen, 253:227-232, 1980.

[Ov88] M.L. Overton. On minimizing the maximum eigenvalue of a symmetric matrix. SIAM Journal on Matrix Analysis and its Appplications, 9(2):256-268, 1988. 